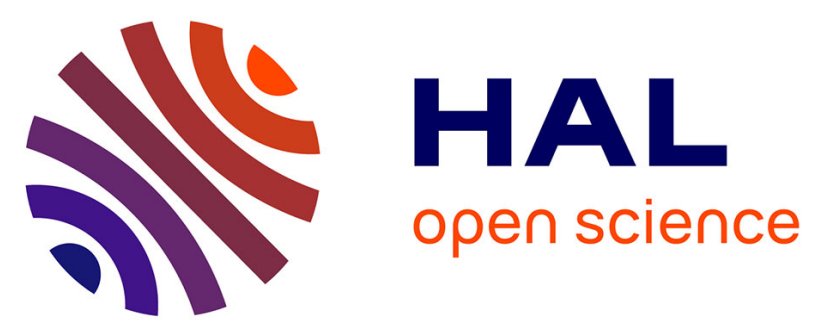

\title{
Magnetic resonance imaging measurements evidence weak dispersion in homogeneous porous media
}

Alizée P. Lehoux, Stéphane Rodts, Pamela Françoise Faure, Eric Michel, Denis Courtier-Murias, Philippe Coussot

\section{To cite this version:}

Alizée P. Lehoux, Stéphane Rodts, Pamela Françoise Faure, Eric Michel, Denis Courtier-Murias, et al.. Magnetic resonance imaging measurements evidence weak dispersion in homogeneous porous media. Physical Review E , 2016, 94 (5), 10.1103/PhysRevE.94.053107 . hal-01784872

\section{HAL Id: hal-01784872}

https://hal-enpc.archives-ouvertes.fr/hal-01784872

Submitted on 3 May 2018

HAL is a multi-disciplinary open access archive for the deposit and dissemination of scientific research documents, whether they are published or not. The documents may come from teaching and research institutions in France or abroad, or from public or private research centers.
L'archive ouverte pluridisciplinaire HAL, est destinée au dépôt et à la diffusion de documents scientifiques de niveau recherche, publiés ou non, émanant des établissements d'enseignement et de recherche français ou étrangers, des laboratoires publics ou privés. 


\title{
MRI measurements evidence weak dispersion in homogeneous porous media
}

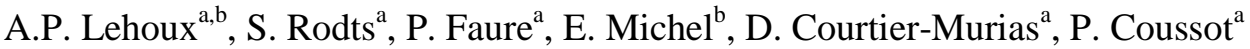 \\ ${ }^{a}$ Université Paris-Est, Laboratoire Navier (ENPC, IFSTTAR, CNRS), Champs-sur-Marne, France \\ ${ }^{\mathrm{b}}$ EMMAH, INRA, Université d'Avignon et des Pays de Vaucluse, Avignon, France
}

\begin{abstract}
We measure the dispersion coefficient through homogeneous bead or sand packings at different flow rates from direct MRI visualizations of the transport characteristics of a pulse of paramagnetic nanoparticles. Through 2D imaging we observe homogeneous dispersion inside the sample, but we show that entrance effects may induce significant radial heterogeneities, which would affect the interpretation of breakthrough curve. Another MRI approach then provides quantitative measurements of the evolution in time of the longitudinal particle distribution in the sample. These data can be analyzed to deduce the coefficient of dispersion independently of entrance effects. The values obtained for this "effective" dispersion coefficient are almost ten times lower than the commonly admitted

values.
\end{abstract}

\section{INTRODUCTION}

In many environmental and industrial situations (ground water flows, chromatography, catalysis, etc) it is necessary to describe and predict the dispersion of solutes or contaminants that move with the flow of water through porous media. Mechanical dispersion, in which a solute or colloid particles in a fluid flowing through a porous medium progressively disperse while diffusion due to thermal agitation is negligible, is a well admitted concept [1-2]. Several theoretical approaches have been developed [2-3], mostly relying on the idea that since the structure is disordered each flowing element will follow a path similar to a random walk around the average velocity, a process leading on long times to Gaussian spreading. Some aspects of the physics of the process are nevertheless questioned [4] and anomalous or non-Fickian dispersion, generally attributed to porous medium heterogeneities, has been often observed $[2,5]$ and is the subject of numerous theoretical developments [6]. It was even recently suggested that non-Fickian dispersion should be the norm [7].

Even for homogeneous systems, i.e. for which the physical properties of representative elementary volumes are homogeneous throughout the sample, the experimental knowledge appears somehow fragile. For similar conditions (material and flow characteristics) the basic parameter describing the process, namely the dispersion coefficient $(D)$, is often not precisely determined [2]. The standard description and quantification of the phenomenon basically rely on the so-called "breakthrough curves", i.e. the time distribution, at the exit, of a solute injected as a step in the sample. This approach does not provide any information on the flow characteristics inside 3D porous medium. In this context existing data for flows through granular packings (of grain diameter $d$ ) apparently fall, with some significant scattering, along a master curve [2]. In the regime of dominant mechanical dispersion this master curve can be represented by $D / D_{m} \approx 4 P e$, where $P e=v d / D_{m}$ is the Peclet number, $v$ the average (interstitial) fluid velocity through pores, and $D_{m}$ the particle diffusion coefficient, as estimated from particle size and Stokes-Einstein relation. Various original advanced approaches, relying on flow inversion experiments [8], particle tracking or scattering in matched-index medium [9], tracer concentration displacement [10] or imaging [11], provided further information on the solute distribution in time which apparently confirmed the above results.

In the other hand ${ }^{1} \mathrm{H}$ NMR (Nuclear Magnetic Resonance), which by means of PFG (Pulse Field Gradient) techniques can directly measure the statistics on molecular displacements in a flow over a given time interval, provides a powerful and precise technique to observe dispersion inside random bead packings. Generally, after travelling a distance of 10 to 20 bead diameters the displacement statistics in the main flow direction exhibits a Gaussian shape reminiscent of some asymptotic dispersion regime [12-18]. Apart from two tests [14], existing 
measurements with this technique generally provided values for $D$ smaller than that associated with the average master curve by a factor 2-3 [19-20], 4-5 [13], or 5-10 [21]. Such difference may be attributed to quite limited explored length scales, so that some macroscale effects responsible for higher macroscale dispersivity may be missed. Besides, from MRI (Magnetic Resonance Imaging) of $\mathrm{D}_{2} \mathrm{O}$ injection in chromatography gel columns a significant band broadening due to column inlet was observed [12], and from an analysis of NMR data, it was suggested that imperfect flow injection could also have a significant impact on the dispersion observed from breakthrough curve experiments [22]. This work also showed that a further analysis of PFG data makes it possible to distinguish intrinsic dispersion, which appears to be in agreement with the theoretical prediction for a random network of capillaries [23], whose resulting $D$ is below the master curve (see above) by a factor about 3.

Thus there is a need to explore dispersion by direct measurements inside the sample over sufficiently large length scales and avoid potential impact of injection. In this work, we used MRI to get information on the internal flow characteristics and precise measurements of the evolution of the longitudinal concentration profile over time. We show that this profile is significantly affected by entrance effects which immediately deform the step radially. As a consequence a specific analysis taking into account this deformation must be set up to properly analyze dispersion. The new direct measurements finally obtained by this means provide effective dispersion coefficient values an order of magnitude lower than usually admitted.

\section{MATERIALS AND METHODS}

\section{A. Materials}

As porous media we used bead or sand packings. Glass beads supplied by Sigma Aldrich were washed twice with 5.8 M hydrochloric acid overnight and rinsed with pure water. Their mean diameter $(d)$ was either 570 or $950 \mu \mathrm{m}$. The size distribution (measured with a laser particle size analyzer) of such beads around these mean values are almost perfectly similar (see [24]) when rescaled by $d$, with a polydispersity coefficient, defined as

$P=\sum_{i} \phi_{i}\left|d-d_{i}\right| / d$ where $\phi_{i}$ is the volume fraction of the beads of diameter $d_{i}$, about $10 \%$ in each case. This means that these beads exhibit a slight polydispersity which precludes any crystalline arrangement in the packing.

The sand was "Fontainebleau sand" sieved between 200-250 $\mu \mathrm{m}$, washed with heated nitric acid (65\%) in a water-bath for 2 hours to dissolve organic matter and oxides, then with a $0.1 \mathrm{M} \mathrm{NaOH}$ solution to neutralize the acid, and finally rinsed with deionised water. Under these conditions the sand grain surface is negatively charged.

Sand or glass beads were carefully introduced in the PMMA column (length $21.5 \mathrm{~cm}$, diameter $5 \mathrm{~cm}$ )) in order to avoid the presence of air bubbles. For this purpose, pure water was added from the bottom of the column in the same time as the porous material, with constant manual stirring of the mixture, in order to homogenize the sample and remove bubbles. Then the material was vibrated to enhance packing and finally the sample was closed with a cover pressed onto its top. This procedure leads to a packing for which relative displacements of grains are unlikely. The porosity of the sample in the column, computed from mass measurement of the different components (grains, water, empty column), ranged from 0.38 to 0.4 for the different samples.

As colloidal particles we used superparamagnetic commercially available particles (Molday ION Carboxyl) which have an iron oxide core and are functionalised with surface carboxyl groups (negatively charged). Their iron content is $1.49 .10^{-20}$ moles of iron per particle, and their density is $1.25 \mathrm{~g} / \mathrm{cm} 3$. Diffusion Light Scattering (DLS) measurements at different concentrations showed that the particle diameter was constant and equal to 30 $\mathrm{nm}$ over $30 \mathrm{~min}$., thus indicating that the particles don't aggregate. For these particles we have $D_{m}=1.47 \times 10^{-11} \mathrm{~m}^{2} \cdot \mathrm{s}^{-1}$. The concentration of particles in water was measured from the iron concentration with 
UV-visible spectroscopy Cary 50 Varian at $500 \mathrm{~nm}$. These small superparamagnetic particles are homogeneously dispersed in water. They do not adsorb on the beads or on the sand grains. Moreover, due to a ratio between the grain size and the particle size between 7500 and 32000, most of particles are situated at very large distance (as compared to their size) from the pore walls and steric effects will certainly be negligible.

\section{B. Experimental set up}

The column containing the grain or bead packing is set up at the center of the magnet (see Figure 1). The column bottom is linked to a peristaltic pump. The liquid (pure water) or the particle suspension is injected from the bottom, and goes out of the sample at the top (see Figure 1), through a pipe (diameter $d_{0}=1.52 \mathrm{~mm}$ ) along the central axis of the sample. The sample is ended at each end by a filter $420 \mu \mathrm{m}$ thick with a pore size of $30 \mu \mathrm{m}$ to avoid any loss of sand grain or glass bead through the pipe. During a steady flow of pure water through the porous medium packing we abruptly replace, for some time, water by a solution of colloidal particles at a concentration of $0.47+/-0.04 \mathrm{Fe} \mathrm{mmol} / \mathrm{l}$ at some distance in the upstream pipe (see Figure 1) then we resume the water injection. The injected volume of suspension, determined by weighting the beaker before and after the injection, varies slightly $( \pm 0.2 \mathrm{ml})$ around $10 \mathrm{ml}$ depending on tests. This volume is equal to $6 \%$ of the total liquid volume in the column. The characteristics of the tests are presented in Table 1.

Due to the flow along the pipe length $(2 \mathrm{~m})$ between the point of injection and the sample entrance there might be some effect of the upstream flow on the (initial) distribution of particle concentration reaching in the sample. Since the flow is laminar ( $\operatorname{Re}=\rho V d_{0} / \mu$ is equal to 70 for a typical velocity of $5 \mathrm{~cm} / \mathrm{s}$, where $V$ the mean velocity in the pipe) the flow is essentially a Poiseuille flow with a parabolic profile $\left(13\left(1-(r / R)^{2}\right)\right.$ in $\left.\mathrm{m}\right)$. This implies that the fluid arriving at the column will be a two-phase flow with a central part made of suspension and an outer part made of pure liquid. During the time needed to reach the entrance there will be a limited exchange between these two phases. Indeed the typical length of diffusion over a time of $40 \mathrm{~s}$ is 17 microns, which is almost one hundred times smaller than the pipe diameter. After the entrance in the column the flow characteristics are complex and we could expect some kind of mixing of these two phases leading to an apparent concentration smaller than the injected concentration. It is likely that there is a mixture of these two phases inside the porous medium at the scale of pore. However, if this effect had an impact on the concentration profile it should be more important for a longer distance of pipe between the injection point and the entrance, but comparing the two series of concentration profiles (inside the sample) obtained for a distance (pipe length between injection point and entrance) of 2 and $5 \mathrm{~m}$ we do not see any difference. A possible explanation is that this effect remains minor as compared to the strong entrance effects (flow enlargement, see main text). Under these conditions we assume that, in the same way, the flow in the pipe after the exit has no impact on the results in terms of breakthrough curve.

For some of these tests we also measured the so-called breakthrough curve. A fraction collector was set up at some distance $(2.9 \mathrm{~m})$ from the exit (see Figure 1) which allows getting fluid samples. Output were sampled regularly (approximately every $8 \mathrm{ml}$ ) and weighted. The iron concentration of each sample was measured from light extinction in visible spectrophotometry at $500 \mathrm{~nm}$, and the breakthrough curves could be drawn from these data. 


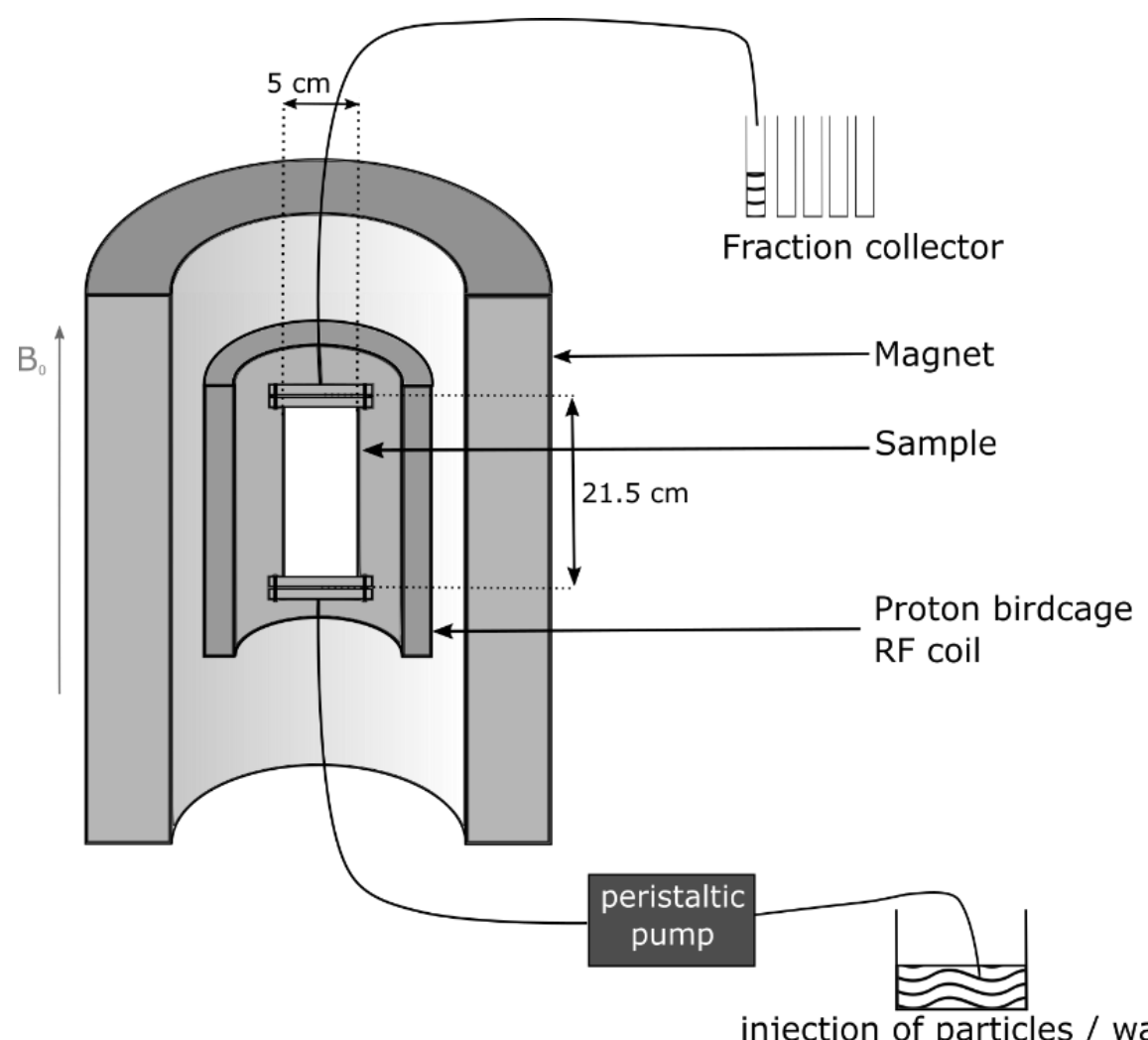

Figure 1: Scheme of principle of the experiment.

\begin{tabular}{|c|c|c|c|c|c|}
\hline & $\begin{array}{l}\text { Average } \\
\text { (interstitial) } \\
\text { velocity in } \\
\text { pores }(\mathrm{m} / \mathrm{s})\end{array}$ & $\begin{array}{l}\text { Dispersion } \\
\text { coefficient } \\
\text { (from } \mathrm{MRI}) \\
D(\mathrm{~cm} 2 / \mathrm{s})\end{array}$ & $D / D_{m}$ & $P e$ & $\begin{array}{l}D / D_{m} \text { from } \\
\text { breakthrough } \\
\text { curve }\end{array}$ \\
\hline \multirow{5}{*}{$\begin{array}{l}\text { Large beads } \\
710-1180 \mu \mathrm{m}\end{array}$} & $4.25 \times 10^{-6}$ & $1.45 \times 10^{-5}$ & 99 & 275 & \\
\hline & $6.9 \times 10^{-6}$ & $3.06 \times 10^{-5}$ & 210 & 447 & \\
\hline & $1.13 \times 10^{-5}$ & $5.35 \times 10^{-5}$ & 367 & 734 & 760 \\
\hline & $1.12 \times 10^{-4}$ & $6.65 \times 10^{-4}$ & 4554 & 7272 & 9389 \\
\hline & $2.23 \times 10^{-4}$ & $1.55 \times 10^{-3}$ & 10641 & 14460 & 17008 \\
\hline \multirow{3}{*}{$\begin{array}{l}\text { Upstream } \\
\text { Downstream } \\
\text { Upstream }\end{array}$} & $6.65 \times 10^{-6}$ & $2.15 \times 10^{-5}$ & 148 & 431 & \\
\hline & $8.02 \times 10^{-6}$ & $3.57 \times 10^{-5}$ & 245 & 519 & \\
\hline & $6.68 \times 10^{-6}$ & $3.11 \times 10^{-5}$ & 213 & 432 & \\
\hline \multirow{4}{*}{$\begin{array}{l}\text { Upstream } \\
\text { Downstream } \\
\text { Upstream } \\
\text { Average } \\
\end{array}$} & $1.07 \times 10^{-4}$ & $6.32 \times 10^{-4}$ & 4327 & 6894 & \\
\hline & $1.2 \times 10^{-4}$ & $8.58 \times 10^{-4}$ & 5876 & 7790 & \\
\hline & $1.09 \times 10^{-4}$ & $7.4 \times 10^{-4}$ & 5072 & 7027 & \\
\hline & & & & 7236 & 7805 \\
\hline \multirow{3}{*}{$\begin{array}{l}\text { Small beads } \\
425-710 \mu \mathrm{m}\end{array}$} & $1.09 \times 10^{-5}$ & $9.16 \times 10^{-6}$ & 63 & 426 & 444 \\
\hline & $1.16 \times 10^{-4}$ & $1.88 \times 10^{-4}$ & 1287 & 4509 & 4230 \\
\hline & $2.23 \times 10^{-4}$ & $3.85 \times 10^{-4}$ & 2634 & 8690 & 8143 \\
\hline Upstream & $6.7 \times 10^{-6}$ & $1.53 \times 10^{-5}$ & 104 & 261 & \\
\hline
\end{tabular}




\begin{tabular}{|c|c|c|c|c|c|}
\hline \multirow{2}{*}{$\begin{array}{l}\text { Downstream } \\
\text { Upstream }\end{array}$} & $8.42 \times 10^{-6}$ & $1.36 \times 10^{-5}$ & 93 & 329 & \\
\hline & $6.8 \times 10^{-6}$ & $1.85 \times 10^{-5}$ & 127 & 266 & \\
\hline \multirow{4}{*}{$\begin{array}{l}\text { Upstream } \\
\text { Downstream } \\
\text { Upstream } \\
\text { Average } \\
\end{array}$} & $1.14 \times 10^{-4}$ & $3.19 \times 10^{-4}$ & 2187 & 4450 & \\
\hline & $1.33 \times 10^{-4}$ & $2.41 \times 10^{-4}$ & 1649 & 5180 & \\
\hline & $1.15 \times 10^{-4}$ & $4.38 \times 10^{-4}$ & 3001 & 4474 & \\
\hline & & & & 4701 & 5200 \\
\hline \multirow{4}{*}{$\begin{array}{l}\text { Upstream } \\
\text { Downstream } \\
\text { Upstream } \\
\text { Average }\end{array}$} & $1.14 \times 10^{-4}$ & $1.65 \times 10^{-4}$ & 1129 & 4439 & \\
\hline & $1.43 \times 10^{-4}$ & $4.94 \times 10^{-4}$ & 3384 & 5591 & \\
\hline & $1.14 \times 10^{-4}$ & $1.49 \times 10^{-4}$ & 1018 & 4468 & \\
\hline & & & & 4833 & 3728 \\
\hline \multirow{4}{*}{$\begin{array}{l}\text { Sand } \\
200-250 \mu \mathrm{m}\end{array}$} & $1.18 \times 10^{-5}$ & $2.16 \times 10^{-6}$ & 15 & 182 & 694 \\
\hline & $1.19 \times 10^{-4}$ & $6.13 \times 10^{-5}$ & 420 & 1832 & 5054 \\
\hline & $2.05 \times 10^{-4}$ & $9.02 \times 10^{-5}$ & 618 & 3158 & \\
\hline & $2.3 \times 10^{-4}$ & $1.35 \times 10^{-4}$ & 922 & 3539 & 11809 \\
\hline \multirow{3}{*}{$\begin{array}{l}\text { Upstream } \\
\text { Downstream } \\
\text { Upstream }\end{array}$} & $8.18 \times 10^{-6}$ & $6.52 \times 10^{-6}$ & 45 & 126 & \\
\hline & $9.36 \times 10^{-6}$ & $1.08 \times 10^{-5}$ & 74 & 144 & \\
\hline & $8.2 \times 10^{-6}$ & $7.34 \times 10^{-6}$ & 50 & 126 & \\
\hline \multirow{4}{*}{$\begin{array}{l}\text { Upstream } \\
\text { Downstream } \\
\text { Upstream } \\
\text { Average }\end{array}$} & $1.13 \times 10^{-4}$ & $1.12 \times 10^{-4}$ & 766 & 1737 & \\
\hline & $1.33 \times 10^{-4}$ & $1.38 \times 10^{-4}$ & 948 & 2043 & \\
\hline & $1.14 \times 10^{-4}$ & $1.06 \times 10^{-4}$ & 727 & 1761 & \\
\hline & & & & 1847 & 2786 \\
\hline \multirow{4}{*}{$\begin{array}{l}\text { Upstream } \\
\text { Downstream } \\
\text { Upstream } \\
\text { Average }\end{array}$} & $1.17 \times 10^{-4}$ & $5.19 \times 10^{-5}$ & 356 & 1806 & \\
\hline & $1.54 \times 10^{-4}$ & $1.83 \times 10^{-4}$ & 1256 & 2370 & \\
\hline & $1.21 \times 10^{-4}$ & $7.5 \times 10^{-5}$ & 514 & 1864 & \\
\hline & & & & 2014 & 3122 \\
\hline
\end{tabular}

Table 1: Test parameters and measurements. The relative uncertainty on measured dispersion coefficient is $+/-10 \%$ (see text and appendix).

\section{MRI}

MRI experiments were carried out with a vertical imaging spectrometer DBX 24/80 Bruker operating at 0.5T (20MHz proton frequency) and equipped with a birdcage radio frequency coil of $20 \mathrm{~cm}$ inner diameter. The suspended colloidal particles modify local NMR relaxation times of water molecules depending on their local concentration, and can then be detected or quantified by means of relaxation weighted ${ }^{1} \mathrm{H}$ MRI [25]. 2D Images were taken using 2D NMR slice-selective spin-echo imaging at $T_{E}=6.54 \mathrm{~ms}$, with $T_{R}=300 \mathrm{~ms}$. The chosen field of view was $24 * 10 \mathrm{~cm}$, the matrix size $256 * 64$ pixels, and the slice thickness $10 \mathrm{~mm}$. Fastness constraint of the $2 \mathrm{D}$ imaging procedure prevents variations of signal intensity in such pictures from being strictly proportional to particle concentration.

Quantitative measurements can nevertheless be achieved on 1D "profiles" MRI pictures, where the sample is projected onto its longitudinal axis $x$. The local intensity $S(x)$ of $\mathrm{T}_{2}$-weighted spin-echo profiles is used to estimate average concentration $\langle\phi(x)\rangle$ of particles in $1.56 \mathrm{~mm}$ thick cross-sectional layers according to $S / S_{0}=<\exp \left(-2 \phi R T_{E}\right)>$. Note that instead of a simple spin echo, this MRI protocol uses the second echo of a 
CPMG (Carr-Purcell-Meiboom-Gill) sequence [26] to better compensate field inhomogeneities. In the above equation, $S_{0}$ is the signal intensity of the slice without particles, $R$ the particle relaxivity calibrated from independent tests with particle suspensions, and $T_{E}=3.41 \mathrm{~ms}$ is the CPMG echo time. Here the matrix size of 128 pixels and the recycling delay $T_{R}=10 \mathrm{~s}$. Short $T_{E}$ allows approximating $\langle\phi(x)\rangle=\ln \left(S_{0}(x) / S(x)\right) / 2 R T_{E}$. Even in the limit of short $T_{E}$, the latter formula was indeed found far more reliable than a just a linear approximation in the case of inhomogeneous tracer repartition through the slice. Prior to data analysis, axis distortions in $x$ direction at bottom and top of the sample are calibrated and corrected. Measurements of $<\phi(x)>$ over long experimental times also suffer baseline fluctuations attributed to temperature evolution in NMR coils and electronics on the time scale of few hours. Note that these fluctuations were taken into account and corrected in our analysis (see Section III.B). To sum up, from such measurements, we get the profile of concentration distribution along the sample axis, i.e. the average particle concentration in successive crosssectional layers along the axis $x$.

\section{EXPERIMENTAL RESULTS AND DISCUSSION}

\section{A. 2D Imaging}

2D MRI in a central longitudinal section of thickness $10 \mathrm{~mm}$ provides successive pictures of the spatial particle distribution along the sample axis (see Figure 2). Around the entrance and the exit the images are deformed due to magnetic field heterogeneity but a relevant qualitative analysis of the flow characteristics is still possible: the first images show that the front of particles is not straight. We could check that this shape does not result from the upstream flow in the pipe as it was insensitive to the distance between the sample entrance and the injection point in the pipe (see Section II.B).

Actually this front shape finds a simple explanation: as the fluid initially arrives from the small pipe and enters the porous medium along its central axis $(x)$ the fluid elements which will invade the sample sides have to move radially and thus will initially advance in the longitudinal direction more slowly than fluid elements around the central axis. This effect may even yield dead zones in the corners of the entrance [27], which does not seem to occur in our case. Such effect is inherent to injection in a porous medium and a previous attempt [10] to sort it out by setting the solute as a straight step at the sample bottom was probably unsuccessful because of deformation occurring during flow start. It also seems that any type of grid or chamber positioned just before the entrance in the porous medium cannot solve this problem. Indeed, as long as the flow remains laminar and particle diffusion is negligible at the pore scale, in steady state, the streamlines, whatever their shape, will need to cover larger distances to reach the lateral edges, so that in general a similar effect as that observed here should occur. The only way to rule out this problem would consist in pushing the liquid, and then the suspension uniformly distributed in a cross-sectional layer, with a piston of the same size as that of the sample. However this seems very difficult to achieve in practice. At last note that the front shape as shown in Figure 2 is the general case (see also Figure 3) but it can also be more complex as observed with sand in the two series of MRI pictures taken with this material (see Figure 4). In that case we suspect that the sample could exhibit some slight packing heterogeneity around the entrance which allowed an apparently better initial radial spreading but we have no more precise explanation. Anyway these observations do not affect the analysis and results described below. 


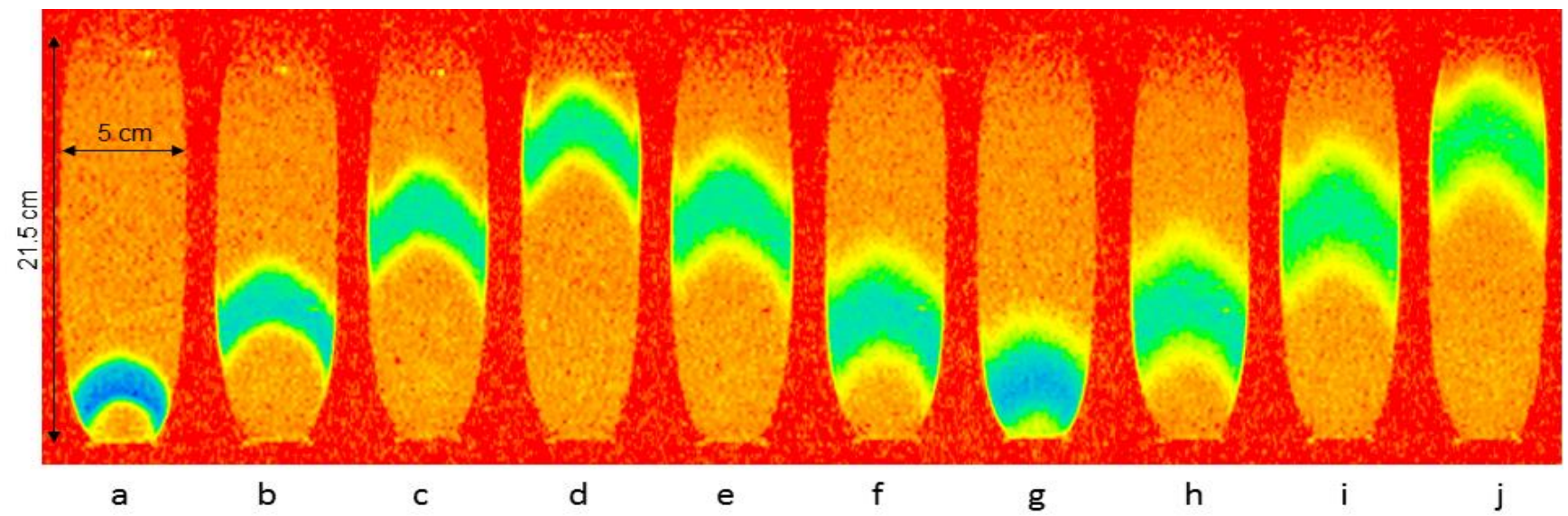

Figure 2: (color online) Images of the particle distribution along longitudinal cross-sections at successive times during the flow through a bead packing $(570 \mu \mathrm{m})$ : first upward flow $(\mathrm{a}, \mathrm{b}, \mathrm{c}, \mathrm{d})\left(v=1.14 \times 10^{-4} \mathrm{~m} \cdot \mathrm{s}^{-1}\right)$, downward flow (e, f, g) $\left(v=1.33 \times 10^{-4} \mathrm{~m} \cdot \mathrm{s}^{-1}\right)$, second upward flow (h, i, j) $\left(v=1.15 \times 10^{-4} \mathrm{~m} . \mathrm{s}^{-1}\right)$. Color scale from maximum concentration to zero: blue-green-yellow-orange. The pictures at top and bottom of the column are distorted due to magnetic field heterogeneity but the sample is cylindrical.

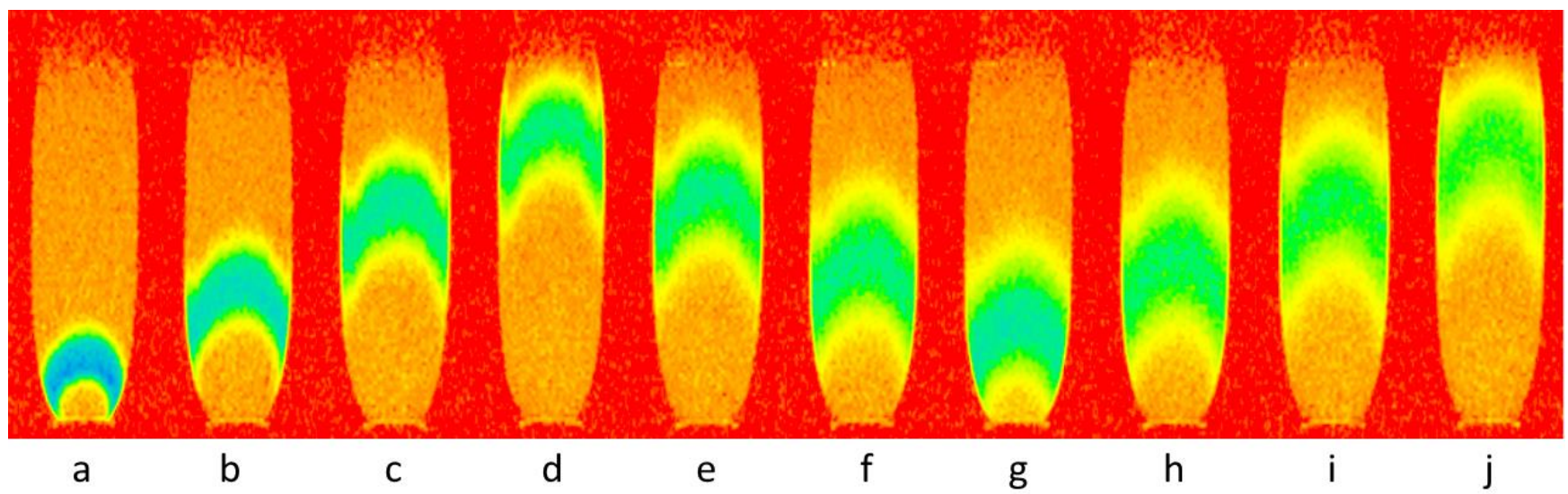

Figure 3: (color online) Images of the particle distribution along longitudinal cross-sections at successive times during the flow through a bead packing $(950 \mu \mathrm{m})$ : upward flow $(\mathrm{a}, \mathrm{b}, \mathrm{c}, \mathrm{d})$, downward flow $(\mathrm{e}, \mathrm{f}, \mathrm{g})$, final upward flow $(\mathrm{h}, \mathrm{I}, \mathrm{j})$. Same scale and colour scale as in Figure 2. First upward flow (a, b, c, d) ( $\left.v=1.06 \times 10^{-4} \mathrm{~m} \cdot \mathrm{s}^{-1}\right)$, downward flow (e, f, g) $\left(v=1.23 \times 10^{-4} \mathrm{~m} \cdot \mathrm{s}^{-1}\right)$, second upward flow $(\mathrm{h}, \mathrm{i}, \mathrm{j})($ $\left.v=1.06 \times 10^{-4} \mathrm{~m} \cdot \mathrm{s}^{-1}\right)$.

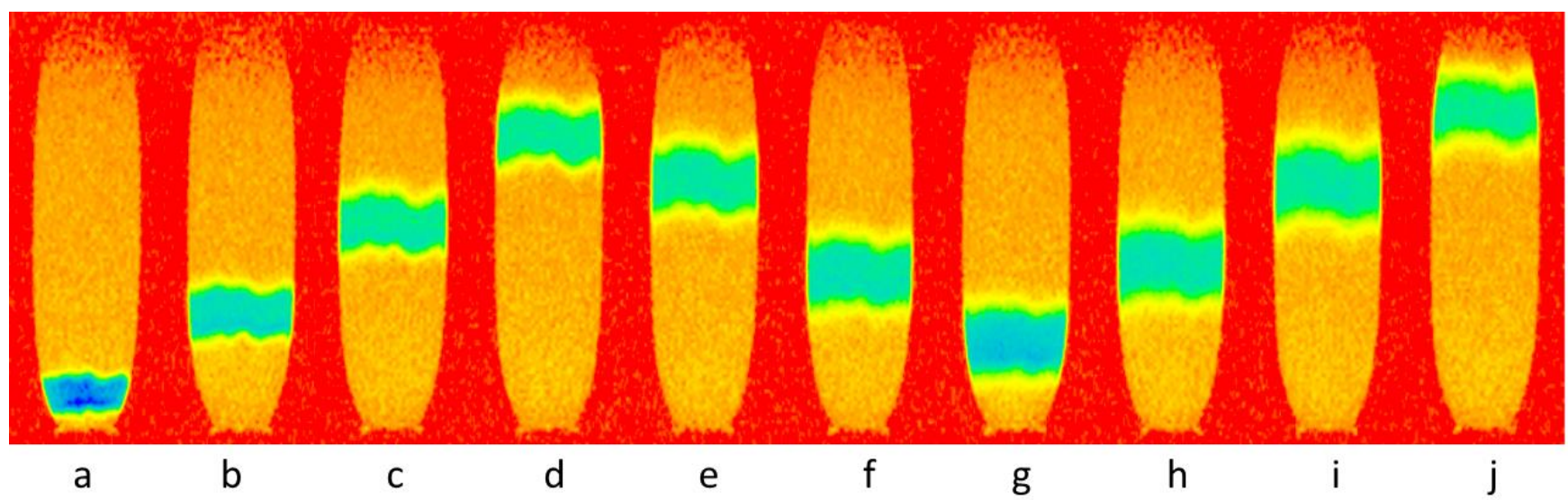


Figure 4: (color online) Images of the particle distribution along longitudinal cross-sections at successive times during the flow through a sand packing $(225 \mu \mathrm{m})$ : upward flow (a,b,c,d), downward flow (e,f,g), final upward flow $(\mathrm{h}, \mathrm{i}, \mathrm{j})$. Same colour scale as in Figure 2. First upward flow (a, b, c, d) $\left(v=1.05 \times 10^{-4} \mathrm{~m} . \mathrm{s}^{-1}\right)$, downward flow (e, f, g) $\left(v=1.21 \times 10^{-4} \mathrm{~m} \cdot \mathrm{s}^{-1}\right)$, second upward flow $(\mathrm{h}, \mathrm{i}, \mathrm{j})\left(v=1.05 \times 10^{-4} \mathrm{~m} \cdot \mathrm{s}^{-1}\right)$.

Finally, after a displacement over a distance approximately equal to the sample diameter the radial shape of the particle distribution in the 2D layer apparently negligibly evolves (see Figures 2,3,4): it essentially spreads longitudinally but its curvature remains constant. A more precise analysis confirms this statement. The front shape, simply taken as the apparent outer limit of the yellow regions, was determined from the successive pictures such as those shown in Figures 2,3,4. The corresponding curves are plotted in Figure 5 for data of Figure 2. The curves superimpose very well within the noise on measurement, it is not possible to detect any trend of evolution of the global shape of the profile, whatever the flow direction and the position of the pulse in the sample.

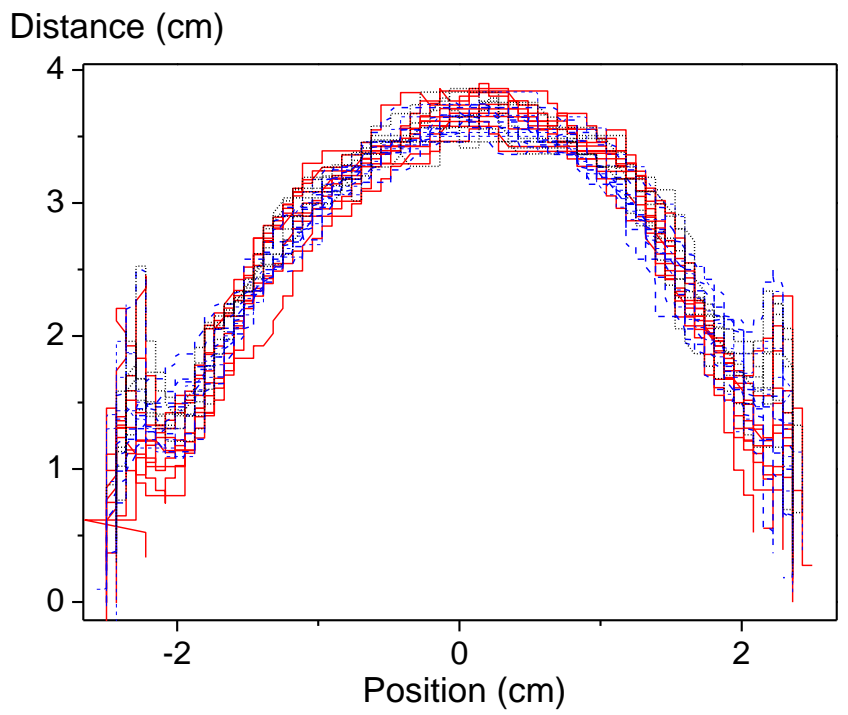

Figure 5: (color online) Successive shapes of the front during pulse displacement in the sample for the test shown in Figure 2: first upwards motion (red continuous), downward motion (blue dashed), second upward motion (black dotted).

These observations mean that on average:

i) the particles essentially move longitudinally;

ii) their average velocity in this direction is now uniform;

iii) the longitudinal dispersion, which was already at work during the entrance stage, is now the major effect.

These conclusions are confirmed by further tests. If we now reverse the flow direction just before the particle front reaches the sample top we do not see any further significant evolution of the radius of curvature of the front (see Figure 2,3,4 d,e,f,g) and this is the same for the next flow reversal (see Figures 2,3,4 g,h,i,j). Thus, as long as the step does not get out of the sample there is no further front deformation but only dispersion. Moreover it is remarkable that there is apparently no impact of gravity and no impact of the flow reversal (indeed the dispersion coefficient was independent of the flow direction, see below). This shows that the dispersion in that regime and over a length much larger than the grain size is fully irreversible, confirming the previous observation of Rigord et al. [28] from ionic tracer echo dispersion measurement. Since the transverse dispersion coefficient was 
observed, either by standard techniques or by NMR [13], to be more than ten times smaller than the longitudinal dispersion coefficient, we will neglect it here. Actually this assumption is consistent with our 2D observations, in particular the fact that the front shape remains apparently constant when moving three times through the sample (see Figures 2,3,4 then Figure 5) whereas significant transverse dispersion with such a curved pulse front (associated with a transverse particle concentration gradient) would tend to progressively deform the front.

There is nevertheless an effect which deserves to be mentioned. In the pictures (especially Figures 2 and 3) we can see a spike along the wall, essentially visible on the "left" side, whose height grows as the particle pulse advances. It has been suggested that the dispersion is enhanced due to radial heterogeneities in bead packing such as those resulting from the wall proximity, even for rather large ratios between the column diameter and the bead diameter [29-30]. However in our case this ratio is of the order of 1/100, so that this effect should be minor. Moreover here this spike has two remarkable characteristics: (i) its growth rate is larger than that of the pulse width, so that it reaches a larger height than the central pulse front at the approach of the column end (see Figures $2 \mathrm{~d}$ and $3 \mathrm{~d}$ ); (ii) this spike tends to disappear during the reversal flow (see Figure 2,3 e,f,g), and then again significantly grows during the last upward flow (see Figure 2,3 h,i,j). This means that, in contrast with dispersion (see above), this effect is almost fully reversible and tends to develop more widely in the flow direction. Such an effect is likely due to some inhomogeneity of the sample along the wall, inducing a slightly smaller solid volume fraction and a larger permeability allowing locally higher velocity. Nevertheless, due to the limited resolution of these 2D pictures, it is not relevant to try to further quantify this effect from our data. As a consequence we will not take it into account in the data treatment below but we can keep in mind that this effect tends to slightly enhance the apparent dispersion, and thus give slightly larger values for the dispersion coefficient than for a flow through a perfectly homogeneous sample.

As already mentioned, fastness constraint of the $2 \mathrm{D}$ imaging procedure prevents the quantitative conversion of signal intensity in such pictures to particle concentration. We can nevertheless get a qualitative view of the concentration distribution along the sample axis by integrating the signal, obtained in these 2D images, in each elementary cross-section. The resulting total signal associated with each position gives a profile of the concentration distribution along the longitudinal direction. From the successive pictures along the main axis we get a series of profiles (see Figure 6) whose height slightly decreases while their width increases. Dispersion is thus already apparent.

\section{B. 1D imaging}

\section{Concentration profiles}

From the 1D imaging procedure we can get a quantitative description of the evolution of the particle distribution along the sample axis. Typical results (see Figure 6) show a good consistency between these profiles and those deduced from imaging (which as for them were extracted from 2D images). Differences owe to shifted timings for $1 \mathrm{D}$ and $2 \mathrm{D}$ measurements, lack of quantitativity of $2 \mathrm{D}$ data, and the fact that unlike 1D profiles, 2D measurements do not probe the whole sample section. This confirms that the shape of the 1D profiles is essentially determined by the step deformation at the entrance and then dispersion can tend to further spread them. 


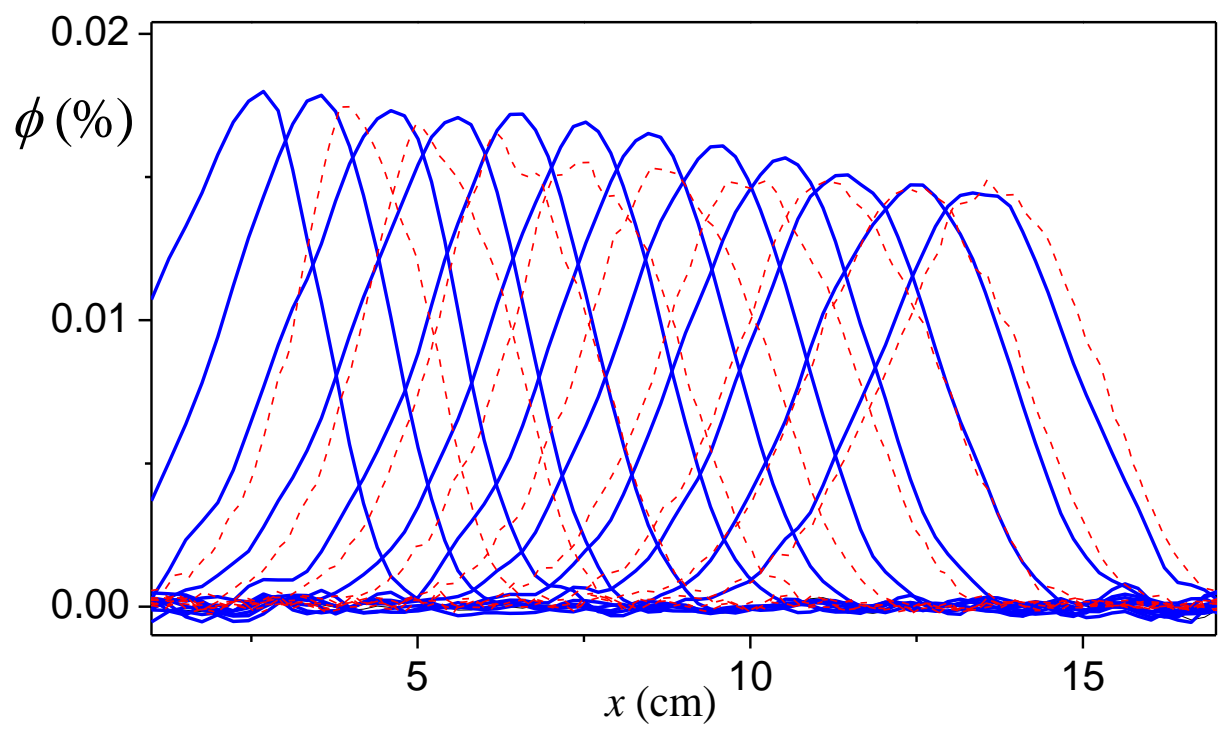

Figure 6: (color online) Longitudinal 1D profiles (continuous blue lines, corrected from baseline fluctuations) of paramagnetic volume particle concentration $(\phi)$ at different times during the first upward flow (every 89 s) for the test of Figure 1. Profiles (dashed red lines) computed from 2D images (see text) (every $106 \mathrm{~s}$ ). For these profiles the concentration scale (assumed to be proportional to the grey level) was arbitrarily chosen so as to get a level for the initial profile close to that from 1D profile at the same instant.

Qualitatively similar results are obtained for different materials (sand packing and glass bead packing) with mean grain diameters from 220 to $950 \mu \mathrm{m}$, different step volumes and average fluid velocities from $1.2 \times 10^{-5} \mathrm{~m} \cdot \mathrm{s}^{-1}$ to $2.4 \times 10^{-4} \mathrm{~m} \cdot \mathrm{s}^{-1}$. This corresponds to Peclet ( $P e$ ) numbers between 200 and 20000 (see Table 1), a range in which mechanical dispersion is dominant [2]. In order to illustrate the range of aspects of concentration profile evolutions during the flow through the column due to the fluctuations from one test to another, we show three additional typical examples, corresponding to approximately the same Peclet number but different resulting values of dispersion coefficient (see Table 1). To better show the profile shape evolution we shifted them of the displacement expected from the mean flow velocity recorded (see Figure 7).

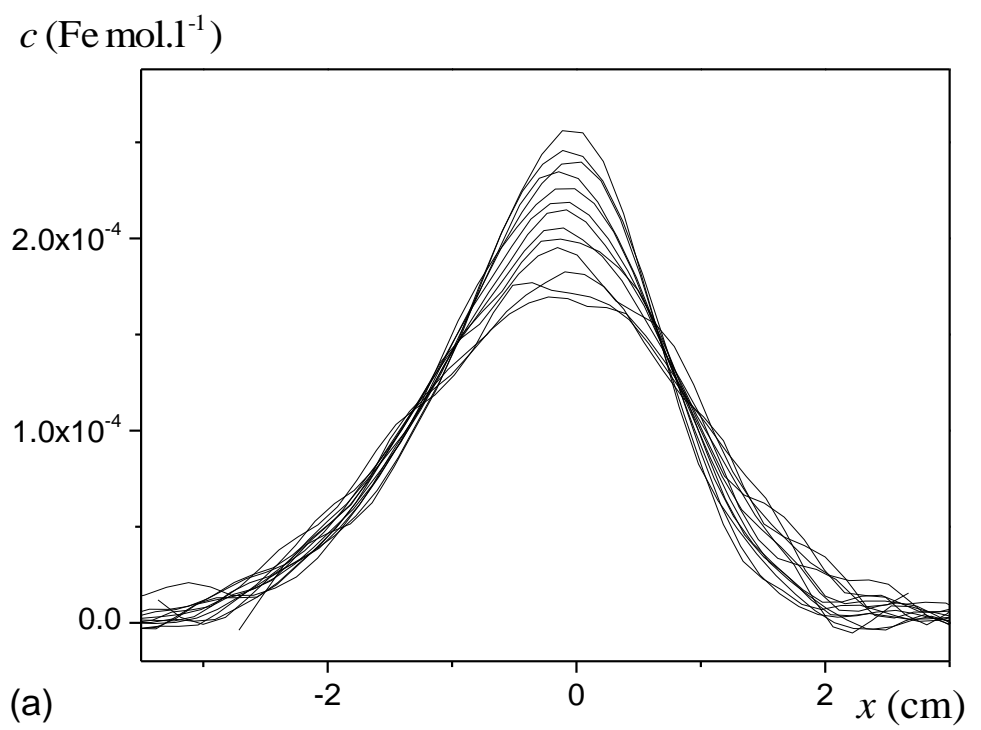



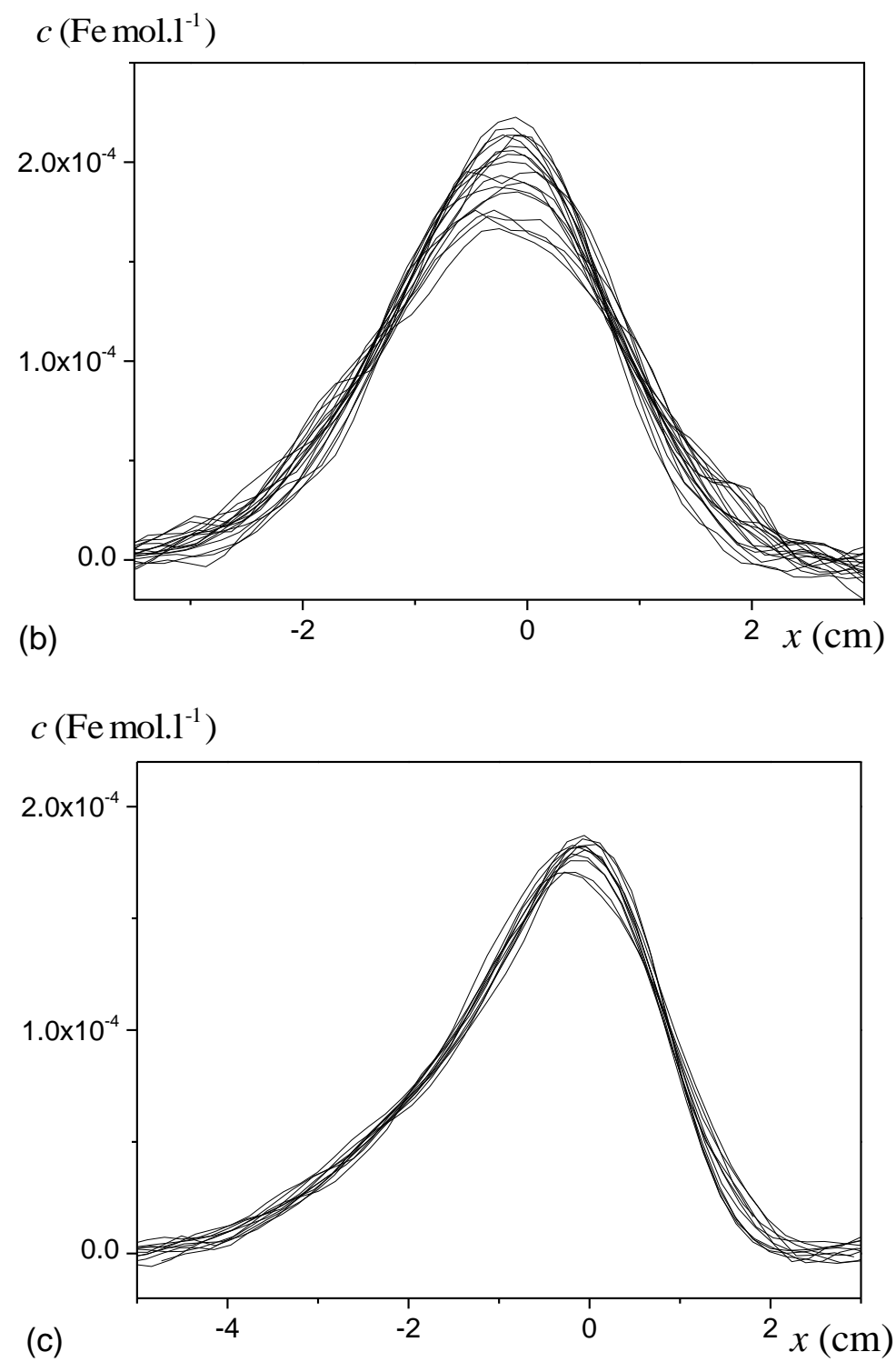

Figure 7: Shifted (see text) concentration profiles for different tests with close Peclet number values: (a) large beads with $P e=447$ (measured $D / D_{m}=210$ ); large beads with $P e=431$ (measured $D / D_{m}=148$ ); small beads with $P e=426$ (measured $D / D_{m}=63$ ). The volume fraction of particles is equal to $c$ times $0.9471 . \mathrm{Fe} \mathrm{mol}^{-1}$.

\section{Analysis of dispersion}

As the 2D images do not provide full quantitative information on the dispersion inside the whole sample we will rely on the 1D profiles. However, since these profiles are generally strongly impacted by the heterogeneity of the 2D distribution of colloids, the analysis of dispersion from such profiles has to take this into account. In that aim we assume that initially the particle concentration in water is of no particular shape due to uncontrolled entrance effects. Regions occupied by the suspension are supposed to be distributed in the form of longitudinal fingers of some constant characteristic thickness and progressing at the same velocity $v$ along sample axis. In the sample let $s_{f}$ be the cross section area of a given finger $f$, and $F_{f}(x)$ the local tracer concentration in this finger at $t=0$ (see Figure 8). 


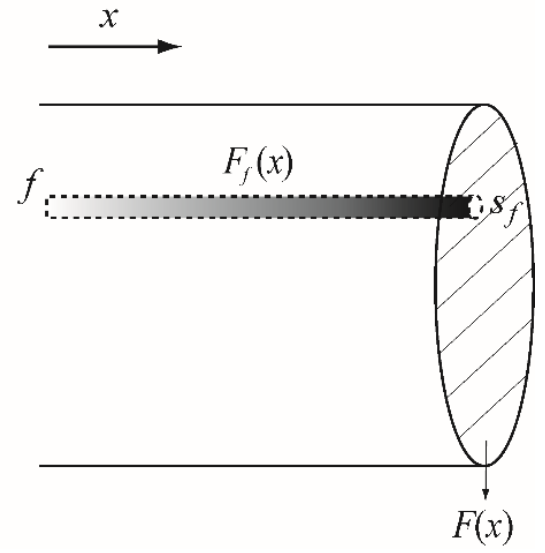

Figure 8: Scheme of the principle of calculation described in the text.

The global concentration profile at $t=0$ then writes $F_{0}(x)=\sum_{f} F_{f}(x) s_{f}$. Assuming that each finger independently spreads as a result of velocity $v$ and standard Gaussian dispersion in $x$ direction, the new profile at time $t$ writes:

$F(x, t)=\sum_{f}\left[F_{f}(x) * g(x, t)\right] s_{f}=F_{0}(x) * g(x, t)$

where $*$ is a convolution product along $x$ direction, and $g(x, t)=\exp \left(-(x-v t)^{2} / 4 D t\right) / \sqrt{4 \pi D t}$ is the 1D dispersion propagator. $F(x, t)$ then still obeys the $1 \mathrm{D}$ equations for Gaussian dispersion. The dispersion coefficient $D$ is determined from a least square fit to the whole data set, where the initial concentration profile $F_{0}(x), \quad v$ and $D$ are taken as fitting parameters. Note that the important parameters sought for in this analysis are transport parameters $D$ and $v$ only, $F_{0}(x)$ is deduced from the fitting procedure and does not need to be measured directly, and a precise definition of time origin is not required, provided it is taken after the pulse enters the sample, and before the first profile is measured. Note that we could check that the fitted velocity was close to the value measured in the hydraulic network. The fitting procedure is described in detail in Appendix 1.

This approach applied to each of our tests well fitted the data within experimental noise level, and provides the longitudinal dispersion coefficient with a maximum uncertainty of $10 \%$ (error bars covered by symbols in Figure 9). 


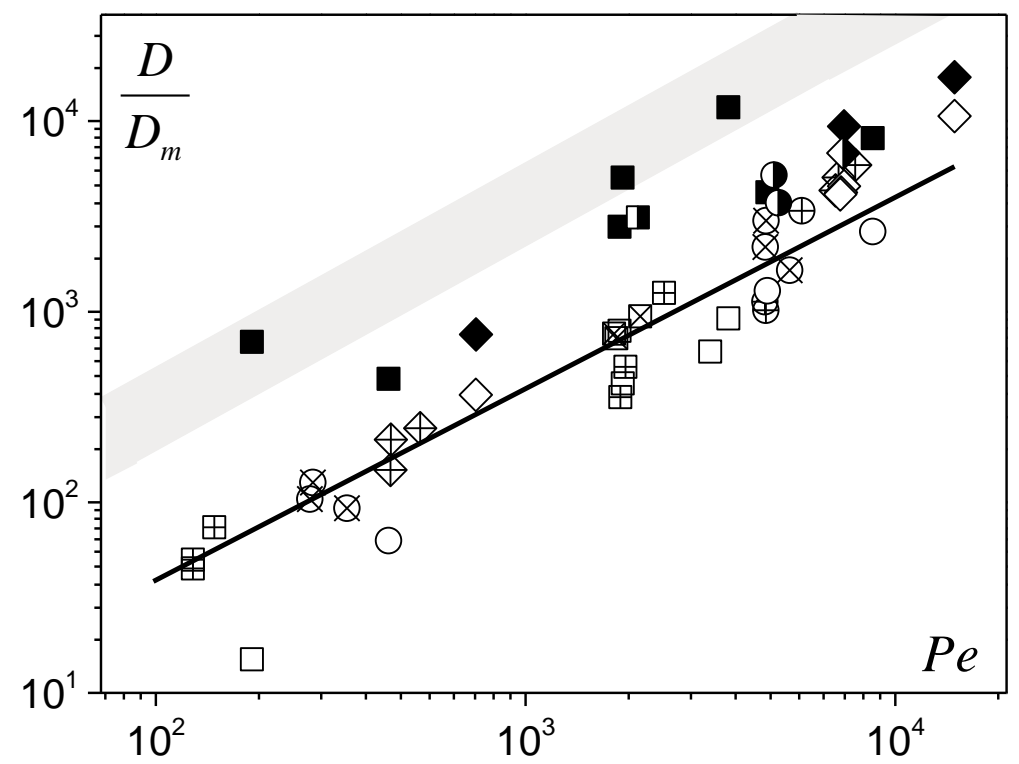

Figure 9: Dispersion coefficient scaled by the diffusion coefficient of the particles computed from the 1D NMR profiles for the different tests as a function of the Peclet number for the different materials: sand (squares), small beads (circles), large beads (diamonds). Crossed symbols correspond to tests with a sequence of upstream-downstream-upstream flows with the same particle step. The grey area represents the region covered by previous data (by conventional techniques) as gathered in Figure 1 of [16], which covers the data leading to the "universal" flow curve as for example shown in [1] and various other data obtained more recently. Dispersion coefficients determined from breakthrough curves are represented by filled symbols (single flow) and half-filled symbols (two flow reversals). The continuous line is equation (2).

The values for the dispersion coefficient obtained by this approach for all our tests are shown in Figure 9. No specific difference is observed for the different material types (grain size, narrow or wide grain size distribution): in logarithmic scale the dispersion coefficient falls along a straight line with a significant scattering. We have no clear explanation for this scattering: it is usual to consider that it is due to fluctuations in the detailed characteristics of the sample structure from one sample to another; however, depending on experiments, sequences of tests with successive upstream and downstream flows through the same sample may exhibit either no difference at all in the dispersion coefficient (superimposed crossed symbols) or some significant variation from one flow to another of the order of that which may be observed for different samples (see Figure 9), suggesting that other effects occur. These effects might be due to slight velocity heterogeneities in a crosssection not taken into account in our modeling.

Under these conditions it is very instructive to estimate the dispersion coefficient via the standard procedure, i.e. from the breakthrough curve. We find values which are significantly scattered and, on average, situated slightly below those generally shown in literature (see Figure 9). These values obtained from breakthrough curves are finally significantly larger than those deduced from internal measurements by a factor of about 4 . This result is consistent with our observation that the shape of the concentration profile is mainly governed by entrance effects. Thus the breakthrough curve results from this initial deformation, then some dispersion in the sample, and finally a possible further deformation at the exit since the fluid elements the farthest from the central axis will reach the exit later than those situated close to this axis. As a consequence the impact of entrance and exit effects can a priori be minimized by using a sufficiently long sample. This is indeed what we observe from measurements of dispersion coefficient from breakthrough curves after two flow reversals, and thus a longer path, inside the column: the resulting coefficient values are smaller and thus closer to the values determined from internal observations (see Fig.9). 
Finally the dispersion coefficients obtained by a direct analysis of the evolution of the concentration distribution inside the sample, and a priori not affected by any artefact, are significantly smaller (on average by a factor about 10) than values obtained so far from standard experiments (see Fig.9). As a first approximation we can represent these data by the following simple function:

$$
D / D_{m}=0.4 P e
$$

This tends to confirm the lower than previously admitted dispersion coefficient deduced from NMR PFG experiments at the length scale of a few tens of glass beads (see Section I). Note that it has been suggested that the Schmidt number (i.e. ratio of kinematic viscosity to diffusion coefficient) has an impact on dispersion [3132]. According to these references the dispersion coefficient is larger for higher Schmidt number, but the correlation suggested in this publication does not go beyond a Schmidt number of 2000. If we nevertheless extrapolate qualitatively this result to our situation which corresponds to a Schmidt number of 54000, we can conclude that our experiments should provide larger dispersion coefficients than usually found, which tend to reinforce our conclusion on the discrepancy between our data and usual data obtained from breakthrough curves.

\section{Conclusion}

By directly monitoring the motion of nano-particles over the sample length, and analyzing for the first time these displacements with a straightforward method insensitive to entrance or exit effects, we find dispersion coefficient on average lower than the data usually serving as reference for engineering (e.g. models predicting pollutant dispersion in environment) by almost an order of magnitude. Previous NMR-PFG measurements over relatively short distances inside the sample also provided lower values of the dispersion coefficient, but in a range slightly above our average value. A possibility is that many previous data based on the standard interpretation of breakthrough curve were affected by entrance and exit effects. This questions the relevance of previous theories attempting to represent these former data. Moreover the fact that dispersion directly measured at much lower values than usually expected in homogeneous systems suggests that it might be a rather difficult task to appreciate the effective "abnormality" of dispersion in other systems.

Acknowledgments: We are grateful to J.P. Hulin for enlightening discussions, and we thank Y. Méheust, T. Le Borgne and R. Juanes for fruitful discussions during the initiation of this work. We acknowledge technical help from B. Jouaud, G. Sevenier and M. Vielpeau from EMMAH.

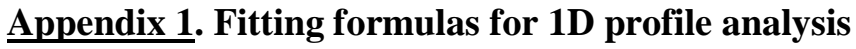

$F_{0}(x)$ is described as a sum of neighbouring positive Gaussian functions following:

$$
F_{0}(x)=\sum_{n} \frac{a_{n}}{\sqrt{2 \pi \sigma_{n}^{2}}} \exp \left(-\frac{\left(x-x_{n}\right)^{2}}{2 \sigma_{n}^{2}}\right)
$$

where $x_{n}$ is a list of about 15 fixed coordinates spanning the initial peak domain with a constant step $d x$. We used $d x=0.5 \mathrm{~cm}$ in all our fits. It was checked that smaller values did not improve fitting procedure, and that changing the interval covered by $x_{n}$ 's did not produce any change in deduced transport coefficients. With such a Gaussian decomposition, model function for $F(x, t)$ is still analytic, following: 


$$
F(x, t)=\sum_{n} \frac{a_{n}}{\sqrt{2 \pi \sigma_{n}^{2}+4 \pi D t}} \exp \left(-\frac{\left(x-x_{n}-v t\right)^{2}}{2 \sigma_{n}^{2}+4 D t}\right)
$$

Due to their specific thermal nature, baseline distortions in our measurements for each measuring time $t_{i}$ are modelled as the product of a fixed baseline shape and a fluctuating coefficient:

$\operatorname{Base}\left(x, t_{i}\right)=\operatorname{Shape}(x) * p_{i}$

where Shape(x) can be modelled as either a 6th order polynomial, or a Fourier expansion truncated to its 7 first terms.

The sum of $F(x, t)$ and $\operatorname{Base}(x, t)$ are fitted to the data, taking as fitting parameters $D, v, a_{n}$ 's, $\sigma_{n}$ 's, $p_{i}$ 's, and polynomial or Fourier coefficients of Shape $(x)$. The fit usually involved about 2000 to 40000 data points, and 100 fitting coefficients. Relevance of the fit was then carefully controlled. First of all, constraints $a_{n} \geq 0$ and $3 d x \geq \sigma_{n} \geq d x / 3$ were used to ensure fitting to be well posed and stable. The fitting algorithm was an iterative constrained trust-region method, and the iteration was stopped when least square criterion could not be further reduced regarding computer double precision accuracy. It was always checked visually that fits well corresponded to data. Residual fitting discrepancy was found to well correspond to noise level on measurements, and this noise level was then used to estimate error bars on fitted parameters. In particular, relative errors on dispersion coefficient never exceeded $10 \%$. It was finally checked that fitted $D$ values did not significantly depend on base-line description, nor on the detailed choice of $x_{n}$ 's -provided they well span the initial peak domain-, nor on small variations of constraints put on $a_{n}$ 's and $\sigma_{n}$ 's, i.e. variations were kept far under the error bar.

\section{References}

[1] A.E. Scheidegger, The physics of flow through porous media (The Macmillan Company, New York, 1960); M. Sahimi, Flow and Transport in Porous Media and Fractured Rock (Wiley-VCH Verlag GmbH \& Co. KGaA, Weinheim, 2011); D. Kulasiri, Non-Fickian solute transport in porous media (Springer, Berlin, 2013)

[2] J. Bear, Dynamics of fluids in porous media (Dover, New York, 1988); F.A.L. Dullien, Porous media - Fluid transport and porous structure (Academic Press, San Diego, 1992)

[3] A.G. Hunt, T.E. Skinner, R.P. Ewing, B. Ghandarian-Alavijeh, Eur. Phys. J. B, 80, 411 (2011); J.H. Cushman, M. Park, M. Moroni, N. Kleinfelter-Domelle, D. O’Malley, Stoch. Environ. Res. Risk Assess., 25, 1 (2011)

[4] C.P. Lowe, D. Frenkel, Phys. Rev. Lett., 77, 4552 (1996)

[5] D. Khandai, D. Hlushkou, A.G. Hoekstra, P.M.A. Sloot, Phys. Rev. Lett., 88, 234501 (2002); P. Gouze, R. Leprovost, T. Poidras, T. Le Borgne, G. Lods, P.A. Pezard, C.R. Geoscience, 341, 965 (2009)

[6] B. Bijeljic, P. Mostaghimi, M.J. Blunt, Phys. Rev. Lett., 107, 204502 (2011); P.K. Kang, M. Dentz, T. Le Borgne, R. Juanes, Phys. Rev. Lett., 107, 180602 (2011); P. de Anna, T. Le Borgne, M. Dentz, A.M. Tartakovsky, D. Bolster, P. Davy, Phys. Rev. Lett., 184502 (2013); B. Berkowitz, H. Scher, Adv. Water Resources, 32, 750 (2009); X. Zhang, M. Lv, Water. Resources Res., 43, W07437 (2007)

[7] J.H. Cushman, D. O’Malley, J. Hydrology, 531, 161 (2015)

[8] J.P. Hulin, T.J. Plona, Phys. Fluids A 1, 1341 (1989); J.P. Hulin, D. Salin, Experimental Methods in the Physical Sciences, 35, 425 (1999)

[9] M. Moroni, J.H. Cushman, Water Resources Res., 37, 873 (2001); M. Moroni, N. Kleinfelter, J.H. Cushman, Adv. Water Resources, 30, 1 (2007)

[10] N.W. Han, J. Bhakta, R.G. Carbonell, AIChE J., 31, 277 (1985)

[11] G. Guillot, G. Kassab, J.P. Huh, P. Rigord, J. Phys. D: Appl. Phys., 24, 763 (1991)

[12] S.G. Harding, H. Baumann, J. Chromatography A, 905, 19 (2001) 
[13] J.D. Seymour, P.T. Callaghan, AIChE J., 43, 2096 (1997)

[14] M.H.G. Amin, S.J. Gibbs, R.J. Chorley, K.S. Richards, Proc. R. Soc. London A, 453, 489 (1997)

[15] S. Stapf, K.J. Packer, R.G. Graham, J.F. Thovert, P.M. Adler, Physical Review E, 58, 6206 (1998)

[16] A.A. Khrapitchev, S. Stapf, P.T. Callaghan, Phys. Rev. E, 66, 051203 (2002)

[17] S. Stapf, S. Han, NMR Imaging in Chemical Engineering (Wiley, New-York, 2006)

[18] B.S. Akpa, D.J. Holland, A.J. Sederman, M.L. Johns, L.F. Gladden, J. Magn. Resonance, 186, 160 (2007)

[19] L. Lebon, J. Leblond, J.P. Hulin, Phys. Fluids, 9, 481 (1997); M. Deurer, I. Vogeler, B.E. Clothier, D.R. Scotter, Transport in Porous Media, 54, 145 (2004)

[20] A.A. Khrapitchev, P.T. Callaghan, Phys Fluids, 15, 2649 (2003)

[21] V. Guillon, M. Fleury, D. Bauer, M.C. Neel, Phys. Rev. E, 87, 043007 (2013)

[22] U. M. Scheven, R. Harris and M. L. Johns, Phys. Rev. Lett. 99, 054502 (2007)

[23] P.G. Saffman, J. Fluid Mech., 7, 194 (1960)

[24] T. Chevalier, C. Chevalier, X. Clain, J.C. Dupla, J. Canou, S. Rodts, P. Coussot, J. Non-Newt. Fluid. Mech, 195, 57 (2013)

[25] E. Keita, P. Faure, S. Rodts, P. Coussot, Phys. Rev. E, 87, 062303 (2013)

[26] H. Carr and E. Purcell, Physical Review 94, 630 (1954); S. Meiboom and D. Gill, Review of Scientific Instruments 29, 688 (1958)

[27] J.L. Paulsen, M.H. Donaldson, S.S. Betancourt, Y.-Q. Song, J. Magn. Resonance 212, 133 (2011)

[28] P. Rigord, A. Calvo, J.P. Hulin, Phys. Fluids A5, 681 (1990)

[29] R.S. Maier, D.M. Kroll, H.T. Davis, AIChE J., 53, 527 (2007)

[30] E. Vandre, R.S. Maier, D.M. Kroll, A. McCormick, H.T. Davis, AIChE J., 54, 2024 (2008)

[31] J.M.P.Q. Delgado, Chemical Engineering Research and Design, 85, 1245 (2007)

[32] J.M.P.Q. Delgado, J.R.F. Guedes de Carvalho, Transport in Porous Media, 44, 165 (2001) 